\title{
Sistema para la medición, visualización y registro de la radiación solar
}

\author{
Ismael Díaz Rangel, María del Consuelo Escoto Ponce de León, \\ Enrique José Tinajero Pérez, Luis Francisco Hernández Villalobos, \\ Gustavo Angel Pioquinto Pérez \\ Universidad Autónoma del Estado de México, Ecatepec de Morelos, \\ México \\ ismael1099@hotmail.com, consuelo_escoto@hotmail.com, ejtinajerop@uaemex.mx, \\ luis123456z114@gmail.com,gustavo_pioquinto@hotmail.com
}

\begin{abstract}
Resumen. La luz solar es esencial para la vida, una exposición moderada a la radiación ultravioleta contribuye a la síntesis de vitamina $\mathrm{D}$, pero exponerse mucho puede ser dañino para la piel, ojos y el sistema inmunitario. Uno de los mayores problemas al exponerse al sol es el cáncer de piel. En México ocurren alrededor de 1,000 casos anuales; los estados con mayor registro de muertes por cáncer son: Ciudad de México, Estado de México, Veracruz y Jalisco [1]. De acuerdo con la doctora Minerva Gómez, Presidenta de la Fundación Mexicana de Dermatología (FMD), este tipo de cáncer ocupa el segundo lugar en frecuencia en nuestro país: el primero en el caso de los hombres, y el segundo respecto a las mujeres [2]. La exposición directa al sol es una amenaza para todas las personas, de manera particular para los menores de edad, por ello es importante contar con medidas preventivas oportunas. La idea de este proyecto es proporcionar a las personas información en tiempo real de la radiación solar a la que se exponen, esto mediante dispositivos que permitan medir el nivel de radiación ultra violeta (UV) y mostrar visualmente la mediación. La propuesta realiza mediciones con múltiples sensores, y mediante un algoritmo ad-hoc mejora el nivel de precisión, el algoritmo también determina el correcto funcionamiento de sensores, haciendo al sistema más confiable; adicionalmente se guarda un registro de las mediciones, lo cual podrá ser útil para análisis a largo plazo. Todo es implementado usando dispositivos de bajo costo.
\end{abstract}

Palabras clave: IVU, cáncer, sensores, medición.

\section{System for the Measurement, Visualization and Recording of Solar Radiation}

\begin{abstract}
Sunlight is essential for life, moderate exposure to ultraviolet radiation contributes to the synthesis of vitamin D, but much exposure can be harmful to skin, eyes and immune system. One of the biggest problems when exposed to the sun is skin cancer. In Mexico, around 1,000 cases occur annually; the states with the highest record of cancer deaths are: Mexico City, Mexico State, Veracruz y
\end{abstract}


Jalisco [1]. According to Dr. Minerva Gómez, President of the Mexican Foundation of Dermatology (FMD), this type of cancer is the second most common in our country: the first in the case of men, and the second in regard to women [2]. Direct exposure to the sun is a threat to all people, particularly for children, so it is important to have timely preventive actions. The idea of this project is provide to the people real-time information about the solar radiation to which they are exposed, using devices that measure the level of ultraviolet (UV) radiation and visually show the measurement. The proposal makes measurements with multiple sensors, and using an algorithm ad-hoc improves accuracy level, the algorithm also determines the correct functioning of sensors, making the system more reliable. Additionally, a record of the measurements is saved, which may be useful for long-term analysis. Everything is implemented using low-cost devices.

Keywords: UV Index, Cancer, Sensors, Measurement.

\section{Introducción}

Todos estamos expuestos a la radiación UV procedente del sol y de numerosas fuentes artificiales. La región UV abarca el intervalo de longitudes de onda de 100 a $400 \mathrm{~nm}$ y se divide en las siguientes tres bandas: UV-A (315-400 nm), UV-B (280$315 \mathrm{~nm})$ y UV-C (100-280 nm). Cuando la luz solar atraviesa la atmósfera, el ozono, el vapor de agua, el oxígeno y el dióxido de carbono absorben toda la radiación UV-C y aproximadamente el $90 \%$ de la radiación UV-B. La atmósfera absorbe la radiación UV-A en menor medida. En consecuencia, la radiación UV que alcanza la superficie terrestre se compone en su mayor parte de rayos UV-A, con una pequeña parte de rayos UV-B; no obstante, los rayos UV-B son responsables de las quemaduras de sol y el cáncer de piel [1].

En el ser humano, una exposición prolongada a la radiación UV solar puede producir efectos agudos y crónicos en la salud de la piel, los ojos y el sistema inmunitario. Las quemaduras solares y el bronceado son los efectos agudos más conocidos de la exposición excesiva a la radiación UV; a largo plazo, se produce un envejecimiento prematuro de la piel como consecuencia de la degeneración de las células, del tejido fibroso y de los vasos sanguíneos inducida por la radiación UV. La radiación UV puede producir también reacciones oculares de tipo inflamatorio, como la queratitis actínica [2].

Se considera que la conducta de las personas con respecto al sol es la razón principal del aumento de las tasas de cáncer de piel en las últimas décadas. El incremento de las actividades al aire libre y los nuevos hábitos al tomar el sol ocasionan a menudo una excesiva exposición a la radiación UV. Además de sus ventajas para la salud, los programas educativos eficaces pueden favorecer las economías de los países, reduciendo la carga financiera que suponen para los sistemas de atención de salud los tratamientos del cáncer de piel y de las cataratas. En todo el mundo se gastan miles de millones de dólares en el tratamiento de estas enfermedades, que en muchas ocasiones se podían haber prevenido o retrasado [3]. 
El IUV es una medida de la intensidad de la radiación UV solar en la superficie terrestre (Figura 1); este índice se expresa como un valor superior a cero, y cuanto más alto, mayor es la probabilidad de lesiones cutáneas y oculares, y menos tardan en producirse esas lesiones.

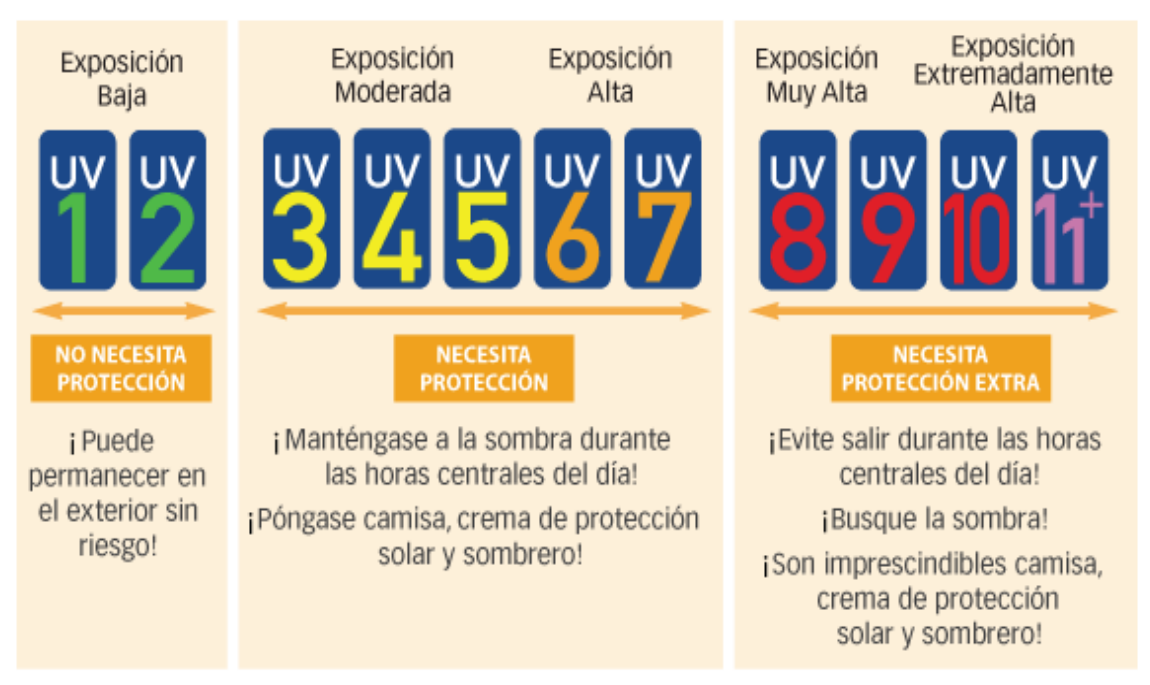

Fig. 1. Escala del índice de radiación solar.

De acuerdo con cifras de la Organización Mundial de la Salud, el cáncer de piel es el más frecuente entre los seres humanos y cada día se diagnostican 2 millones de casos en todo el mundo [4].

En palabras de la doctora Minerva Gómez, Presidenta de la Fundación Mexicana de Dermatología (FMD), este tipo de cáncer ocupa el segundo lugar en frecuencia en nuestro país: el primero en el caso de los hombres, y el segundo respecto a las mujeres [5]. Es importante considerar que es necesario sensibilizar a la población e incitar la toma de medidas de prevención, como es la protección solar particularmente en niños; por lo cual, este proyecto propone crear un sistema simple, fiable y económico de proporcionar información en tiempo real de los niveles de radiación solar a la que nos exponemos, y con eso motivar a tomar medidas de protección, esperando disminuir los casos de cáncer en la piel. Se podría pensar que los niveles de radiación UV nos son tan altos en sitios como en la Ciudad de México, sin embargo hay días en los que se llega a registrar el nivel 11, el cual es el considerado el más alto en la escala UV.

Ya existen trabajos relacionados [6], [7] y [8], esta propuesta se diferencia en que las mediciones realizadas son almacenadas en una memoria MicroSD en intervalos de cinco minutos, los cuales pueden ser recuperados para observarse y analizarse en cualquier software de hojas de cálculo; junto con el nivel de radiación se almacena la hora y fecha exacta de la medición, gracias a que se incorpora un reloj de tiempo real. Además, mediante comunicación inalámbrica por radiofrecuencia, el nivel de radiación es enviado (hasta un máximo de $1 \mathrm{~km}$ de manera ideal) a un display, dando la posibilidad 
de implementar el sistema en escuelas y cualquier espacio público para que la gente conozca, en tiempo real, el nivel de radiación al que se expone al realizar actividades al aire libre, y así tomar medidas preventivas adecuadas.

\section{Metodología}

El diseño de la propuesta está conformado por dos subsistemas: Emisor (fig. 2), realiza lecturas de los cinco sensores de radiación UV que incorpora, lectura de la hora y fecha, almacenamiento de la información en una MicroSD y transmisión inalámbrica; Receptor (figura. 4), recibe la información y la muestra en el display que incorpora. Microcontroladores realizan todas las funciones lógicas necesarias.

\subsection{Emisor}

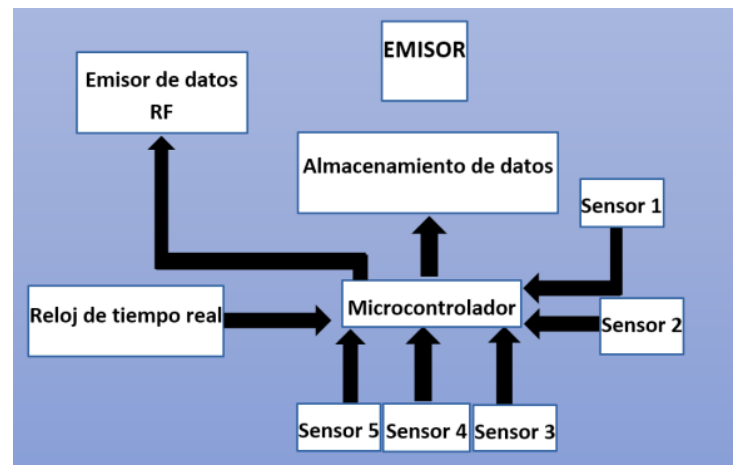

Fig. 2. Diagrama a bloques del emisor.

Sensores. - Se utilizaron los sensores ML8511; lo que se indica a continuación es realizado por cada uno de los cinco sensores: el microcontrolador realiza ocho lecturas consecutivas, con un breve intervalo entre cada una, se obtiene el promedio y se guarda en una variable.

Reloj de tiempo real (RTC). - Se utilizó el DS13307; este bloque lee del RTC la fecha y hora, la información es almacenada en una variable.

Almacenamiento de datos. -La información de fecha, hora y nivel de radiación es almacenada en una memoria MicroSD, utilizando un MicroSD Shield.

Emisor de datos. - Mediante el transreceptor RF NRF24L01, se transmite la información del índice de radiación UV utilizando el standard de radiofrecuencia a 2.4GHZ.

Microcontrolador Atmega328.- Adicionalmente a la generación de señales de control para los diferentes módulos del sistema, se realiza la decodificación las lecturas de los sensores para transfórmalas en el formato de IVU; además, incorpora un algoritmo para determinar y descartar los sensores que están operando de manera fallida; el algoritmo también cuenta con un método que permite obtener una medición 
con precisión mejorada, utilizando la información de los sensores que operan correctamente.

El diagrama esquemático del sistema emisor se muestra en la figura 3.

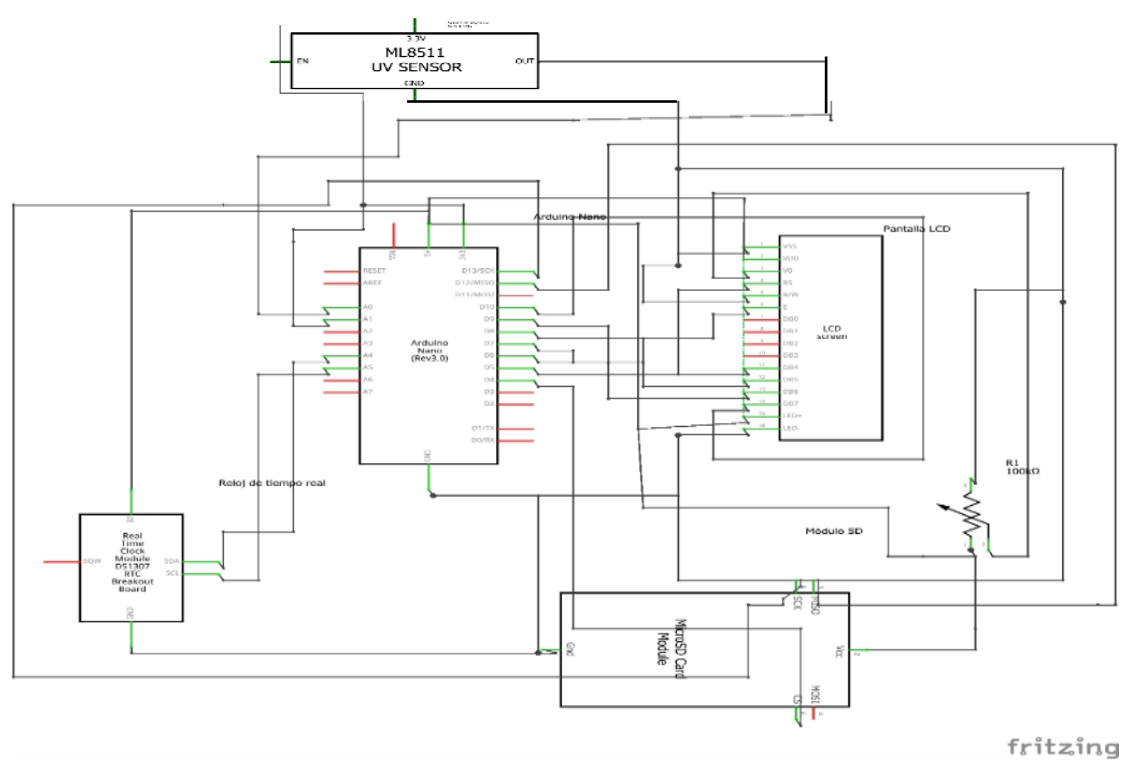

Fig. 3. Diagrama esquemático del emisor.

\subsection{Receptor}

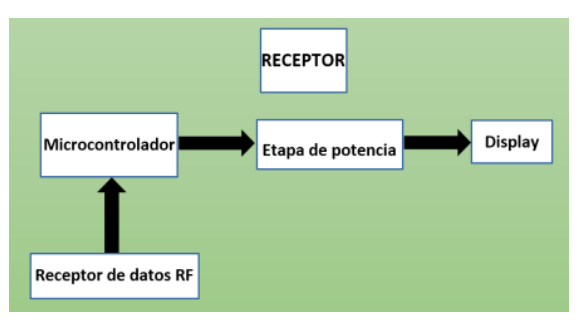

Fig. 4. Diagrama a bloques del receptor.

Receptor de datos. - El microcontrolador realiza una lectura al NRF24L01, en caso de tener un dato se guarda en un registro.

Etapa de potencia. - El microcontrolador decodifica el dato recibido correspondiente al nivel de radiación, y lo transmite en formato de siete segmentos hacia arreglos de transistores en Darlington, poniéndolos en corte o saturación.

Display. - A la etapa de potencia se conectan tiras de LED, las cuales encienden o se apagan de acuerdo el número que representará al nivel de radiación.

En la figura 5 se presenta el diagrama esquemático del Receptor. 
Ismael Díaz Rangel, María del Consuelo Escoto Ponce de León, Enrique José Tinajero Pérez, et al.

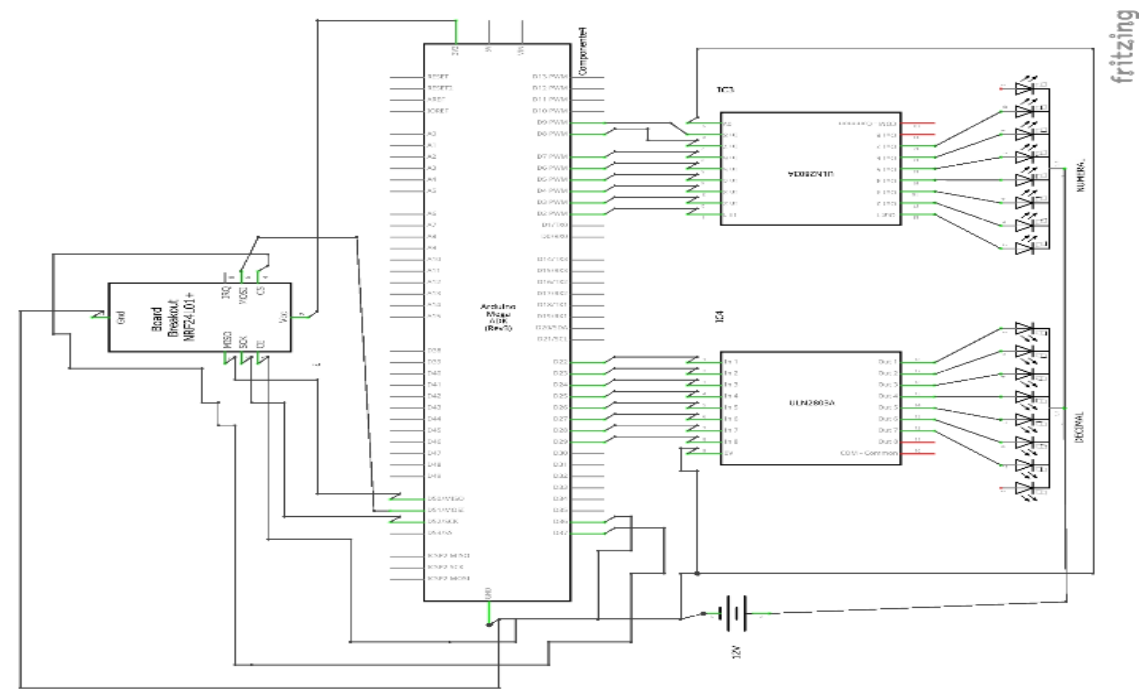

Fig. 5. Diagrama esquemático del receptor.

A continuación, se presenta en la figura 6 el diagrama de flujo del emisor:

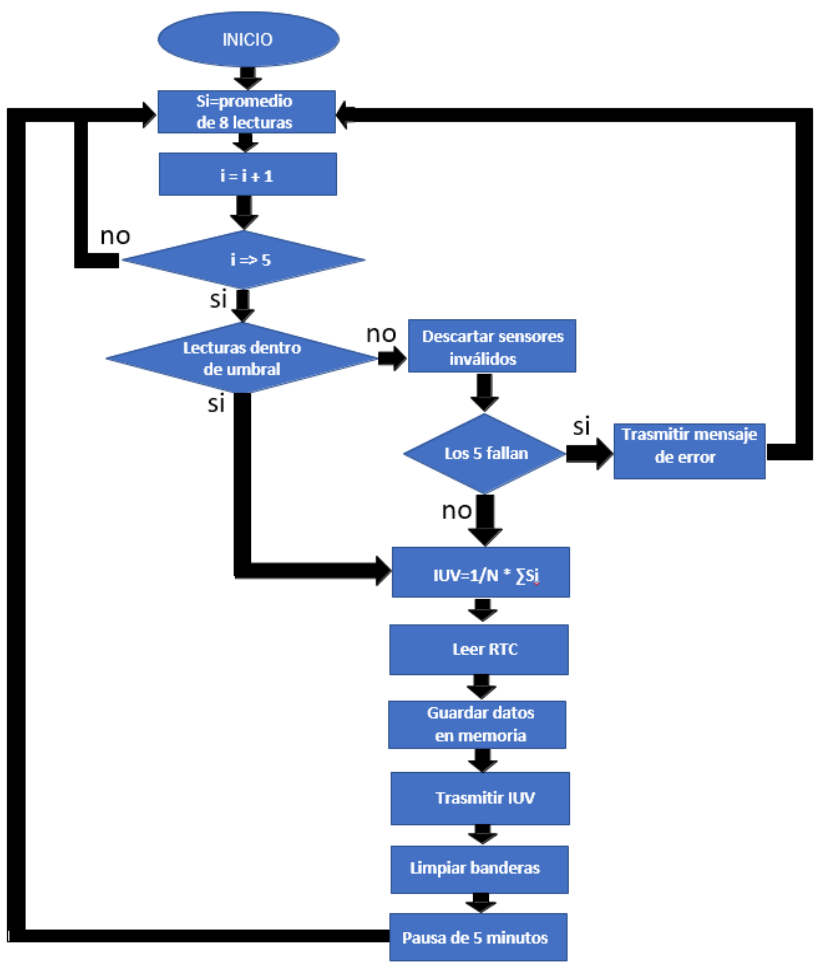

Fig. 6. Diagrama de flujo del emisor. 
Se estableció realizar ocho lecturas consecutivas de cada sensor con un intervalo de $100 \mathrm{~ms}$ entre cada una; posteriormente se promedian las mediciones, y el resultado se guarda en una variable (una para cada sensor); esto tiene la finalidad de reducir el margen de error propio del sensor, mejorando así la precisión del resultado. Para determinar los valores mínimos y máximos que proporcionan los sensores, se realizó el siguiente procedimiento:

Primero, se determinó el valor mínimo que entrega el sensor (cuando no hay radiación UV en el entorno), el resultado se obtuvo con la ecuación 1:

$$
\mathrm{V}_{\text {mínimo }}=\frac{\operatorname{LecSen} * 3.3}{\operatorname{LecSenRef}}
$$

donde:

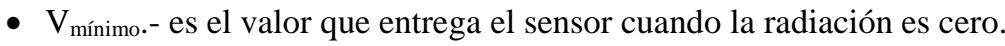

- LecSen.- es el resultado del ADC del microcontrolador al realizar una lectura del sensor.

- LecSenRef.- es el resultado del ADC al realizar una lectura del sensor sobre el voltaje de referencia $(3.3 \mathrm{~V})$.

Para conseguir el valor máximo se aplicó una radiación UV de intensidad nivel 15, y se hizo el procedimiento anterior, arrojando los siguientes resultados:

$$
\begin{aligned}
& V_{\text {mínimo }}=0.99, \\
& V_{\text {máximo }}=2.8 .
\end{aligned}
$$

Estos valores indican que cuando la radiación aplicada al sensor es cero, este entrega 0.99 volts, y cuando la radiación es nivel 15, entrega 2.88 volts. En la hoja de datos del sensor, el fabricante indica que trabaja de manera lineal, por lo que la ecuación que modela su funcionamiento ideal se determinó con la ecuación 2:

$$
I U V=m * \text { LecSen }+b,
$$

donde:

- IUV.- índice de radiación ultravioleta.

- m.- pendiente de relación lineal.

- b.- ordenada al origen.

Para calcular la pendiente (m) se usó la fórmula de la ecuación 3:

$$
m=\frac{\text { Índice de radiación máximo }}{\text { Vmáximo }- \text { Vmínimo }} \text {. }
$$

Sustituyendo los valores obtenidos en los procedimientos anteriormente mencionados, se tiene:

$$
m=\frac{15-0}{2.8-0.99}=8.29 \text {. }
$$

Para la obtención de b, se hace un despeje de la ecuación 2, teniendo: 


$$
b=I U V-m * \text { LecSen. }
$$

$\mathrm{Al}$ sustituir con valores conocidos se tiene:

$$
b=15-(8.29 * 2.8)=-8.2 .
$$

Utilizando la información obtenida con estos experimentos, se pueden descartar sensores que se encuentren en mal estado, y esto será cuando en sus lecturas se observe un voltaje inferior a 0.99 o superior a 2.88 , lo cual fue utilizado en el algoritmo para identificar sensores en mal estado.

Una vez identificados los sensores con funcionamiento correcto ( $\mathrm{N}$ sensores), se procede a obtener el promedio de sus mediciones. Si los 5 sensores están dañados se envía al Display un mensaje de error (letra "e" en código de 7-segmentos); adicionalmente, hay 5 LED, uno para cada sensor, que en caso de mal funcionamiento se envía una señal para que el usuario sepa de dicha situación y proceda a su revisión.

En caso de que cuatro o los cinco sensores operen correctamente, se descartará el de mayor y el de menor resultado, promediando los restantes; esta acción tiene como finalidad mejorar la precisión de las mediciones. El resultado será codificado a formato de 7-segmentos y transmitido al módulo de RF, Para los demás casos se realizarán las mismas acciones pero sin eliminar datos mínimos y máximos.

Adicionalmente, se accede al RTC para obtener los datos de fecha y hora; posteriormente, se almacenan mediante el módulo MicroSD. A continuación, se presenta en la figura 6 el diagrama de flujo del emisor:

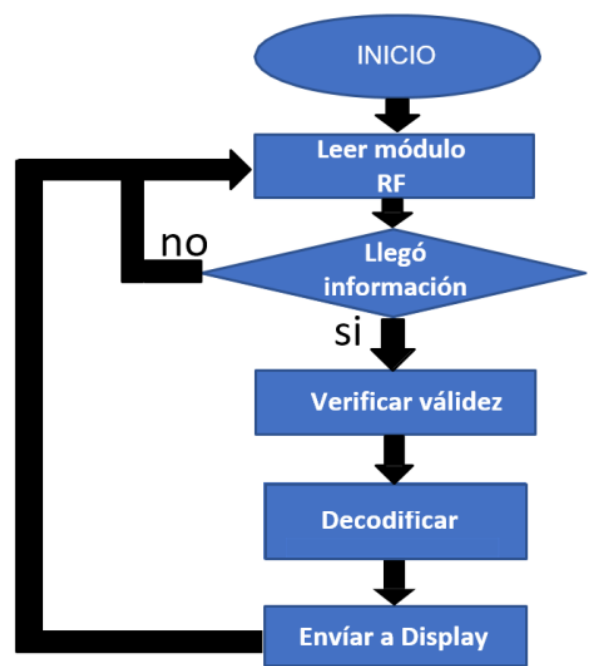

Fig. 7. Diagrama de flujo del receptor

En este subsistema se inicia revisando si llegaron datos prevenientes del Emisor, se verifica su validez; de ser positiva, se procede a decodificar la información para su envío al display de 7-segmentos. 


\section{Resultados}

Para saber la precisión de los sensores, se realizaron múltiples mediciones de manera simultánea a los cinco sensores, se utilizaron lámparas UV a una distancia de $5 \mathrm{~cm}$; en la tabla I muestran los resultados de la prueba.

Tabla 1. Resultados de lecturas aplicando una radiación UV constante.

\begin{tabular}{ccccccc}
\hline \multirow{2}{*}{ Sensor } & \multicolumn{2}{c}{ Muestra 1 } & \multicolumn{2}{c}{ Muestra 2 } & \multicolumn{2}{c}{ Muestra 3 } \\
& Lectura & IUV & Lectura & IUV & Lectura & IUV \\
\hline 1 & 233 & 1.24 & 236 & 1.36 & 237 & 1.41 \\
2 & 236 & 1.36 & 238 & 1.45 & 244 & 1.69 \\
3 & 235 & 1.32 & 236 & 1.36 & 239 & 1.49 \\
4 & 242 & 1.61 & 247 & 1.81 & 245 & 1.73 \\
5 & 233 & 1.24 & 229 & 1.08 & 233 & 1.24 \\
\hline
\end{tabular}

La figura 8 corresponde a la gráfica de la tabla 1, donde se aprecia claramente el alto nivel de precisión de los sensores.

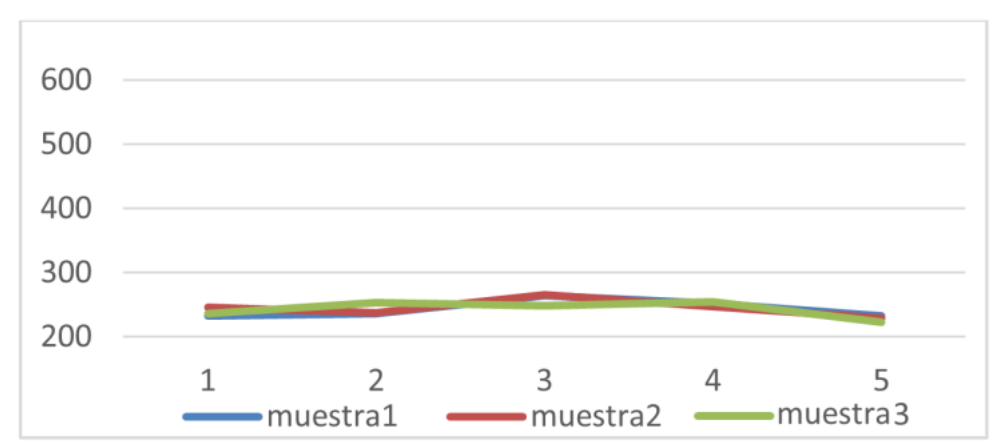

Fig. 8. Gráfica con mediciones de los cinco sensores.

Se realizaron más prueba aplicando diferentes niveles de radiación; la tabla 2 corresponde a la aplicación de un menor nivel de radiación.

Tabla 2. Resultados de lecturas aplicando una radiación UV constante.

\begin{tabular}{ccccccc}
\hline \multirow{2}{*}{ Sensor } & \multicolumn{2}{c}{ Muestra 1 } & \multicolumn{2}{c}{ Muestra 2 } & \multicolumn{2}{c}{ Muestra 3 } \\
& Lectura & IUV & Lectura & IUV & Lectura & IUV \\
\hline 1 & 216 & 0.55 & 217 & 0.59 & 220 & 0.72 \\
2 & 212 & 0.39 & 216 & 0.55 & 215 & 0.51 \\
3 & 212 & 0.39 & 212 & 0.39 & 218 & 0.64 \\
4 & 216 & 0.55 & 209 & 0.27 & 212 & 0.39 \\
5 & 210 & 0.31 & 218 & 0.64 & 216 & 0.55 \\
\hline
\end{tabular}


La figura 9 es la gráfica de la tabla II, donde se aprecia nuevamente el alto nivel de precisión de los sensores.

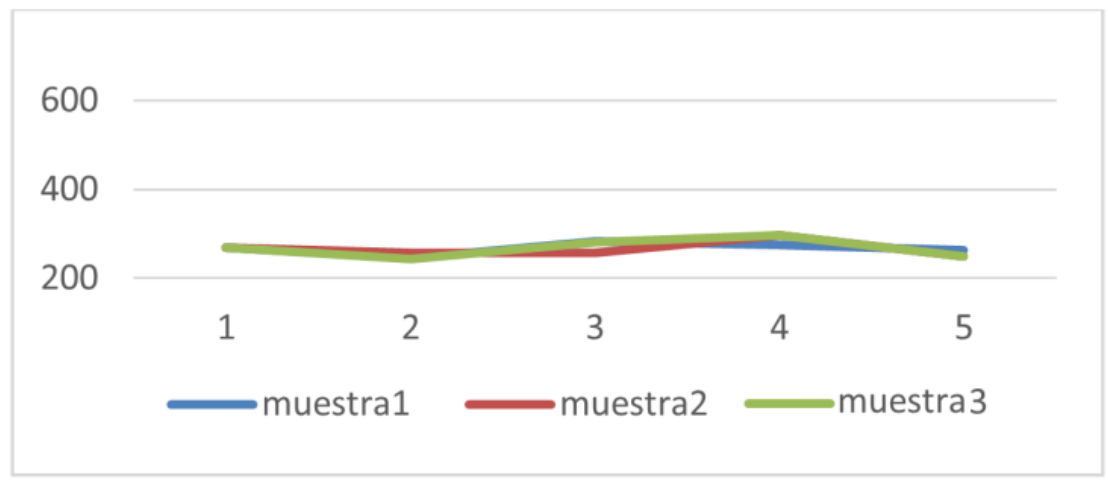

Fig. 9. Gráfica con mediciones de los cinco sensores.

En las dos pruebas presentadas, se aprecia claramente el alto nivel de precisión con que los sensores ML8511 realizan las estimaciones de radiación UV. Adicionalmente se hizo una prueba comparativa con un sistema de medición de la radiación UV profesional, el cual pertenece al SIMAT (Sistema de Monitoreo Atmosférico de la Ciudad de México); para ello se realizaron las peticiones correspondientes a la Secretaria del Medio Ambiente. Para las pruebas se colocó el sistema de esta propuesta a un costado del equipo UV-BIOMETER (Figura 10).

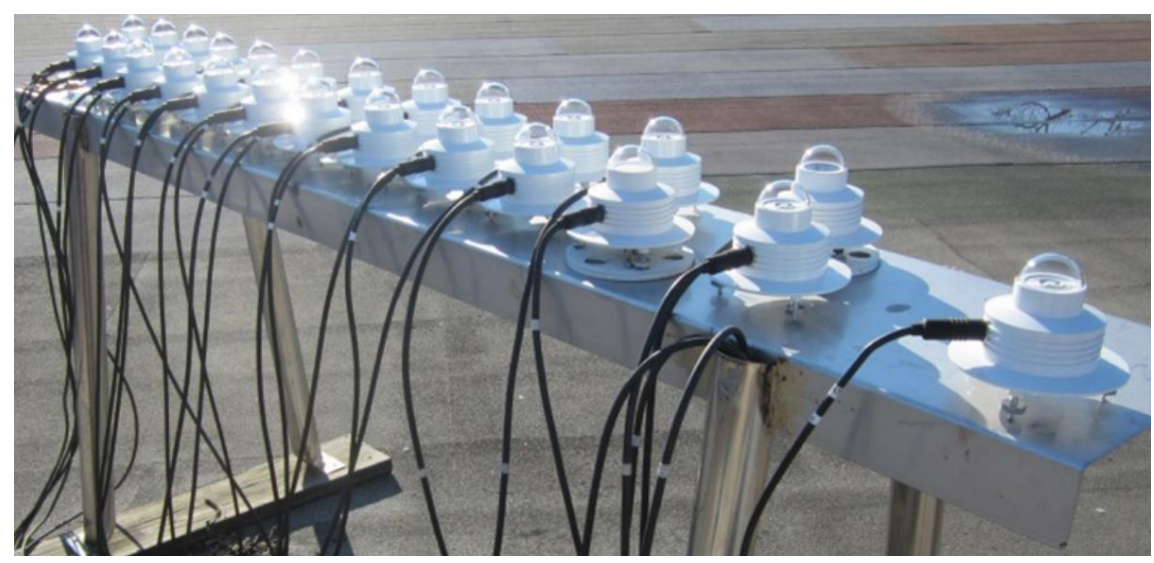

Fig. 10. Radiómetro UV-BIOMETER.

Se tomaron lecturas durante dos días, la figura 11 corresponde a una gráfica comparativa entre ambos sistemas, donde se aprecia claramente el alto nivel de similitud de las mediciones entre ambos sistemas. 
GRAFICA 501 UV-BIOMETER Y SENSOR ML8511

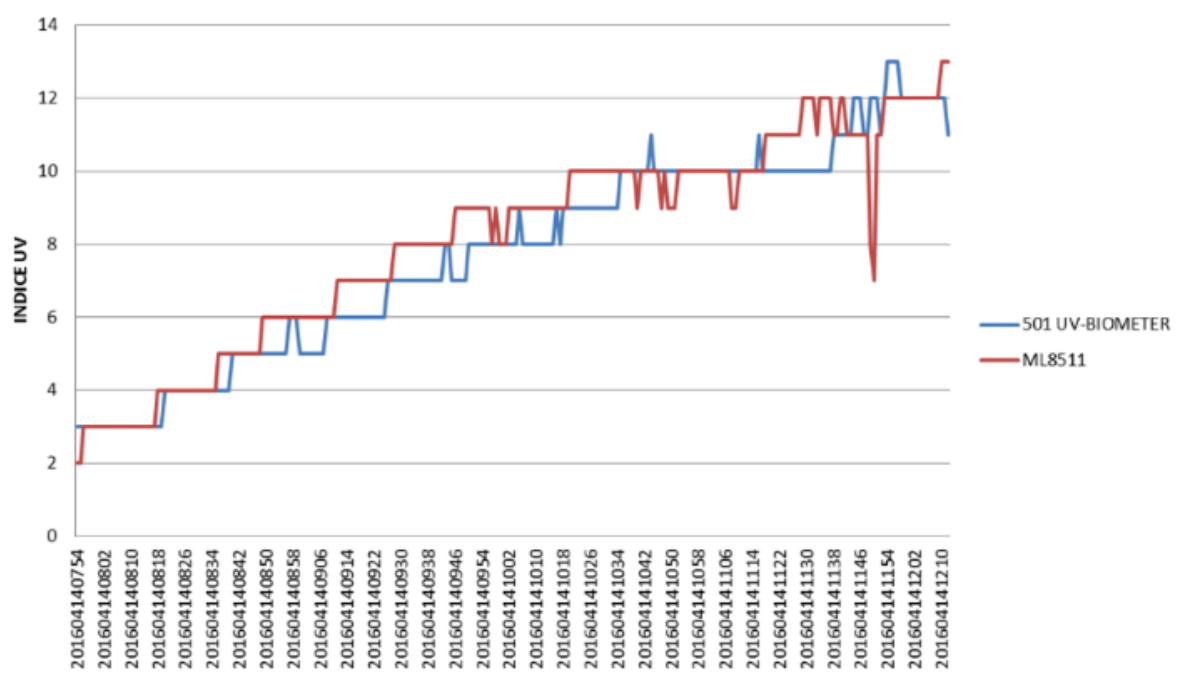

Fig. 11. Gráfica comparativa de mediciones ML8511 vs UV-BIOMETER.

\section{Conclusiones}

En la investigación realizada para conocer los efectos de las radiación UV sobre las personas se pudo constatar, que si bien es beneficiosa para los organismos, una sobre exposición provoca efectos negativos en la salud; desafortunadamente cada año se reportan más casos de cáncer en la piel, siendo este tipo de cáncer el más común en mujeres y el segundo en hombres, estas cifras México.

En la revisión del estado del arte se encontraron varias propuestas, pero ninguna consiste en un sistema que utilice varios sensores para mejorar la precisión, ni de algoritmos para descartar/indicar problemas de funcionamiento de los mismos. Otra importante diferencia, es que uno de sus subsistemas realiza las mediciones, y apoyándose en un reloj de tiempo real y un módulo MicroSD guarda un histórico de las lecturas cada cinco minutos, y que las mediciones son transmitidas inalámbricamente por radiofrecuencia al segundo subsistema, el cual recibe la información, codifica y la envía a un display; se cuenta con una etapa de potencia que permitió usar tiras LED para tener la capacidad de incorporar un display de gran tamaño, lo cual permite su visualización a distancia, haciéndolo apto para su instalación en espacios "abiertos" como patios de escuelas y parques.

Una de las ventajas más importantes de esta propuesta, es que el nivel de precisión es cercano a radiómetros profesionales, pero costando una pequeña fracción en comparación.

Las pruebas realizadas mostraron de manera satisfactoria el correcto funcionamiento de todos los elementos que conforman a esta propuesta, quedando como trabajo futuro agregar una carcasa que permita su fácil instalación y protección a la intemperie. 


\section{Referencias}

1. Arulmozhi, B., Mekala, K., Sivasankari, A.: Storage-Less and Converter-Less Energy Harvesting using Internet of Thinking Technology. International Research Journal of Advanced Engineering, pp. 36-40 (2016)

2. Bienestar180: Recuperado de http://www.salud180.com/salud-dia-dia/cancer-de-piel-creceen-mexico (2017)

3. Diffey, B.L.: Sources and measurement of ultraviolet radiation. Methods, pp. 44-13 (2002)

4. LAPIS Semiconductor: Recuperado de http://www.lapis-semi.com/en/semicon/ sensor/ml8511.html (2016)

5. OMS Organización Mundial de la Salud: Recuperado de http://www.who.int/ mediacentre/news/notes/2005/np07/es/ (2016)

6. Paredes, C. Ibrahin, P.: Diseño de un sistema de señalización utilizando sensores fotovoltaicos para la prevención de los efectos de la radiación solar para los estudiantes de la EPIE. Universidad Nacional del Altiplano (2017)

7. Pérez, T. M.: Diseño de un radiómetro ultravioleta, para su aplicación en modelos de radiación UV. México D.F., UNAM (2014)

8. Sanz, A. Ozono, L.: Recuperado de http://www.pionero.cu/sites/default/files/especiales/ sitios/ozono /cuidadoconsol-22feb09.htm (2016)

9. Riveros, T.: Efectos de la radiación ultravioleta en el ser humano, aspectos clínicos. Comisión de fotobiología de Sochiderm (2016) 